\title{
Features of dynamics of the load-carrying body of the belt tubular conveyor
}

\author{
Tetiana Zhyhula, ${ }^{1, *}$ \\ ${ }^{1}$ Institute of Geotechnical Mechanics named by N. Poljakov of National Academy of Sciences of \\ Ukraine, 49005, Dnipro, Simferopolska Str., 2a, Ukraine
}

\begin{abstract}
The problems of researching the dynamics of the load-carrying body of a belt tubular conveyor (BTC) are formulated. It is noted that for BTC, at which the flat belt is rolled into a pipe with the help of special roller supports, the main danger is torsional vibrations. The factors causing torsional vibrations of the tubular belt are analyzed. An estimate of the maximum angle of rotation of the belt from the suddenly applied moment is given. A comparison of the angular turns of the tubular belt of the closed and open profiles is made. The relations of the main belt's parameters, which exclude the possibility of its significant rotation and load spills are obtained.
\end{abstract}

\section{Introduction}

Recently, tubular belt conveyors (BTC) have been increasingly used in various industries for moving long distances of bulk cargo.

The advantages of this type of conveyors, compared with belt conveyors of traditional design, include the possibility of transporting fine and hazardous materials along complex curvilinear routes without transfer stations, excluding their spillage and dusting. The main disadvantage of BTC is the use of an special belt, the cost of which is commensurate with the cost of the entire conveyor.

Belt tubular conveyors with the use of special belt are manufactured by Phoenix and Koch (Germany), Metco (Finland), Sicon (Sweden). Rather high capital costs for the manufacture of BTC are offset by low operating costs. The world operating experience of BTC showed that the cost of transporting a unit of load is significantly lower than that of other transport means.

The belt of a tubular conveyor must meet specific requirements - possessing increased elasticity and flexibility to stably maintain the shape of the pipe, to withstand considerable loads at loading and unloading points. In order to choose a tape that meets these requirements, it is necessary to investigate the dynamic processes that occur in a tubular belt with a load during its movement.

The tubular belt, when moving along the rollers of conveyor, makes transverse and torsional vibrations. The main danger is torsional vibrations, which are not limited by anything, and are excited due to the torques that occur when the belt is rolled into a pipe,

\footnotetext{
*Corresponding author: zhigulat@gmail.com
} 
when the conveyor belt is bent, when the support rollers are skewed, and when the belt is unevenly loaded.

These moments can cause a significant angular rotation of the belt, and, consequently, the rotation of its overlapped edges, with the possible divergence of the edges, loss of stability of the internal volume, spillage of cargo, dusting. Preventing the rotation of the tubular part of the belt is an important task.

A significant amount of research on the dynamics of tubular conveyors over the past 10 years has been performed at Moscow State Mining University (Russia) by Galkin V.I., Dmitriev V.G., Sergeeva N.V., Egorov A.P., Ivanov I.Yu., Efimov M.S. etc.

These studies are devoted to the vertical and angular oscillations of the tubular belt, the determination of the critical speeds of movement of the conveyor and the resistance forces moving to along the roller belt [1-3]. Although the amount of research has been done extensively, many important issues of BTC dynamics have not been studied.

In [1], it is noted that in order for the performance of a tubular conveyor belt to be close to that of a conveyor with a grooved belt, the speed of a BTC belt must be of the order of $8 \mathrm{~m} / \mathrm{s}$, which may lead to an increase in the resistance forces to the movement of the belt. In order to study the vertical and rotational oscillations of the tape, the corresponding equations were compiled and formulas for the critical velocities and forces of resistance to the movement of the belt were obtained on their basis.

In article [2], rotational oscillations of a tubular belt are investigated for the case when the conveyor route has a bend. Various ways of turning the route are considered, expressions for the torques acting on the tubular belt are obtained, the case of the loss of the tightness of the belt caused by the torque is analyzed.

\section{Methods}

In the study of the torsion of a tubular conveyor belt on a straight section of the route, we represent it in the form of a tubular rod with one rigidly fixed $(x=0)$ and the other free end $(x=1)$, to which torque $M(\mathrm{~N} \cdot \mathrm{m})$ is applied (Fig. 1).

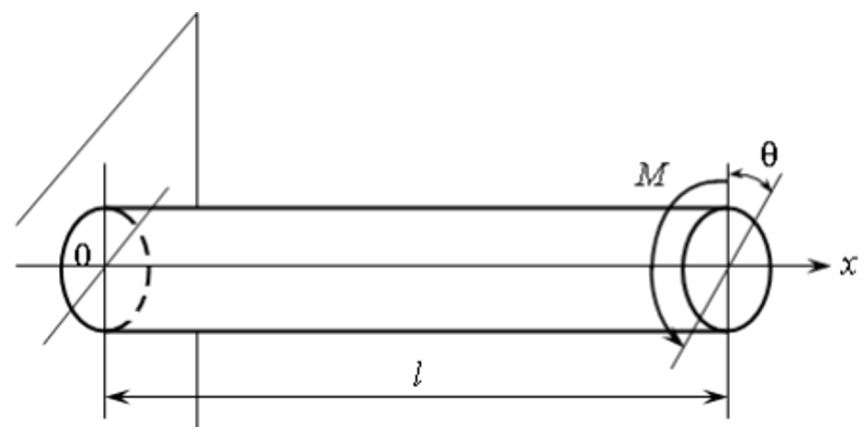

Fig. 1. Design scheme for torsion of a tubular rod.

The total twist angle along $x$, measured from the terminated end, is determined by the integral

$$
\theta=\int_{0}^{x} \frac{M d x}{G I_{p}},
$$

where $G I_{p}$ is the stiffness of the section during torsion, $\mathrm{N} \cdot \mathrm{m}^{2}$.

If the section stiffness $G I_{p}$ is constant throughout the integration area, then 


$$
\theta=\frac{M x}{G I_{p}}
$$

As can be seen from formula (2), as the distance from the fixed end increases, the twist angle increases linearly. The maximum twist angle is obtained at the end of the rod $(x=l)$

$$
\theta_{\max }=\frac{M l}{G I_{p}} .
$$

Formula (3) determines the maximum twist angle of the tubular conveyor belt on the straight part of the route under the action of a time constant moment, which may be due to skewed roller, uneven loading of the belt, etc. Of interest is the study of dynamic phenomena that occur in a tubular belt under the action of a variable in time torque load.

In the study of torsional vibrations of a tubular belt, we represent it in the form of a uniform elastic rod, which is under the action of a moment load intensity $Q(x, t)$ [4]. The differential equation of torsional vibrations of the rod has the form:

$$
\bar{I} \frac{\partial^{2} \theta}{\partial t^{2}}-\frac{\partial}{\partial x}\left[G I_{p} \frac{\partial \theta}{\partial x}\right]=Q(x, t),
$$

where $\theta(x, t)$ - the angle of rotation of the cross section of the rod $x$ at time $t, \mathrm{ra} ; G-$ shear modulus, $\mathrm{N} / \mathrm{m}^{2} ; I_{p}$ - equatorial moment of cross section, $\mathrm{m}^{4} ; \bar{I}$ - linear moment of inertia about the axis of the $\mathrm{rod}, \mathrm{N} \cdot \mathrm{s}^{2}$.

Consider the case of a twisting blow, i.e. the case when the moment $M$ is suddenly applied to the free end of the rod at the initial moment of time.

Then equation (4) takes the form:

$$
\bar{I} \frac{\partial^{2} \theta}{\partial t^{2}}-G I_{p} \frac{\partial^{2} \theta}{\partial x^{2}}=M \sigma_{1}(x-l) \sigma_{0}(t),
$$

where $\sigma_{0}(t)$ - single function; $\sigma_{1}(x-l)$ - impulse function of the first order.

It is necessary to find a particular solution of this equation under boundary conditions

$$
\theta(0, t)=0, \quad\left(\frac{\partial \theta}{\partial x}\right)_{x=l}=0
$$

and under initial conditions

$$
\theta(x, 0)=\dot{\theta}(x, 0)=0
$$

We are looking for a solution in the form of decomposition by proper forms of free torsional vibrations of a rod with one rigidly fixed and one free end

$$
\theta(x, t)=\sum_{k=1}^{\infty} T_{k}(t) \varphi_{k}(x)
$$

where $\varphi_{k}(x)=\sin a_{k} x-$ proper forms;

$$
T_{k}(t)=\frac{2 M \sin a_{k} l}{\bar{I} l p_{k}^{2}}\left(1-\cos p_{k} t\right) ;
$$




$$
a_{k}=\frac{2 k-1}{2 l} \pi ; \quad p_{k}=a_{k} \sqrt{\frac{G I_{p}}{\bar{I}}}, \quad(k=1,2,3, \ldots) .
$$

The final solution sought is

$$
\theta(x, t)=\frac{8 M l}{\pi^{2} G I_{p}} \sum_{k=1}^{\infty} \frac{\sin a_{k} l \sin a_{k} x}{(2 k-1)^{2}}\left(1-\cos p_{k} t\right) .
$$

Putting $x=l$, we find the angle of rotation of the end of the rod

$$
\theta(l, t)=\frac{8 M l}{\pi^{2} G I_{p}} \sum_{k=1}^{\infty} \frac{1-\cos p_{k} t}{(2 k-1)^{2}} .
$$

The maximum angle of rotation of the rod end we get at $\cos p_{k} t=-1$

$$
\theta_{\max }=\frac{16 M l}{\pi^{2} G I_{p}}\left(1+\frac{1}{3^{2}}+\frac{1}{5^{2}}+\ldots\right)=\frac{2 M l}{G I_{p}} .
$$

The maximum angle of rotation of the rod end from a suddenly applied moment turns out to be twice the angle of rotation from the same magnitude of the constantly acting moment.

Let $[\theta]$ be the permissible angle of rotation of the end of the tubular belt, that is, an angle at which no divergence of the sides of the belt and the spilling of the load will occur. Then the condition must be met:

$$
\theta_{\max } \leq[\theta] \quad \text { or } \quad \frac{2 l M}{G I_{p}} \leq[\theta] .
$$

Determining the value of $[\theta]$ and estimating the moment applied to the end of the belt, you can pick up the belt, the basic parameters of which satisfy the last inequality, which will make it possible to eliminate the divergence of the sides of the tubular belt and the load spilling at a sudden application of torque.

The torsional stiffness of the tubular tape $G I_{p}$ depends on the physical properties of the belt material (shear modulus $G$ ) and the geometric characteristics of its cross section (the polar moment of inertia of the cross section $I_{p}$ ). The polar moments of inertia of the sections of solid and cut round thin pipes are determined by the formulas:

$$
\begin{aligned}
& I_{p c}=\frac{\pi}{32}\left(d^{4}-d_{0}^{4}\right) ; \\
& I_{p o}=\frac{\pi}{3}(d-h) h^{3},
\end{aligned}
$$

where $I_{p c}, I_{p o}$ - polar moments of inertia of solid and cut pipes, respectively, $\mathrm{m}^{4} ; d, d_{0}-$ external and internal pipe diameters, respectively, $\mathrm{m} ; h$ - pipe wall thickness, $\mathrm{m}$.

According to (8), (9) and (10), the twisting angles of whole $\theta_{c}$ and cut $\theta_{o}$ pipes of the same size, which are under the action of equal torques, are equal to: 


$$
\begin{aligned}
& \theta_{c}=\frac{64 M l}{\pi\left(d^{4}-d_{0}^{4}\right) G} ; \\
& \theta_{o}=\frac{6 M l}{\pi(d-h) h^{3} G} .
\end{aligned}
$$

\section{Results and discussion}

For real BTC, the ratio of pipe diameter to wall thickness $d / h \geq 20$. For such conveyors, the twisting angle of the cut pipe is 270 times greater than that of a solid pipe with the same torque. The above example confirms the advantage of a tubular belt of a closed profile compared with a tubular belt of an open profile when working in torsion.

We consider BTC, the tubular belt of which is formed by rolling the flat belt into the pipe, that is, the profile of the tubular belt of the conveyor has the form of a ring with a cut. Therefore, in further calculations, we determine the polar moment of inertia of the cross section of the belt using formula (10) and the angle of rotation of the cross section using formula (12). From formulas (8) and (12) we determine the value of the moment from which the angle of rotation of the cross section does not exceed the permissible value:

$$
M \leq \frac{[\theta] \pi(d-h) h^{3} G}{6 l} .
$$

In article [3] it is noted that for BTC with six-rollers supports, when the angle of deviation of the belt is more than $20^{\circ}$, the edge of the belt will come in contact with the adjacent roller, which will lead to increased belt wear and other undesirable phenomena. Based on this, we accept $[\theta]=\pi / 9$, then the relation (13) takes the form:

$$
M \leq \frac{\pi^{2}(d-h) h^{3} G}{54 l} .
$$

Substituting in (14) the parameters of a specific belt, we can determine the limit value of a suddenly applied torque, which does not lead to a significant rotation of the belt.

\section{Conclusions}

1. Studies of the torsional vibrations of the BTC tubular belt showed that the torque applied suddenly to the cross section of the tape causes the section to rotate by an angle twice as large as in the case of static loading with the same moment. Relations have been obtained to determine the basic parameters of the tubular belt, which will exclude the possibility of a significant rotation of the cross section of the tape and the load spilling.

2. The determining parameter for torsional vibrations of a tubular belt is the torsional rigidity of its section $G I_{p}$. The shear modulus of the conveyor belt $G$ is small; the polar moment of inertia $I_{p}$ for a section in the form of a cut ring is also small. Therefore, even small external torque loads can cause significant angular rotations of the section of the tubular belt. When choosing the belt material and the shape of its cross section, it is desirable that the shear modulus of the tape material and the polar moment of inertia of the cross section have as large values as possible. Solid tubular belt is hundreds of times more stable under the action of torques than cut. If this is permissible, it is advisable to use a BTC with a solid tubular belt. 


\section{References}

1. Dmitriev, V. G., Egorov, A. P. (2011). Influence of speed of belt on distributing force of resistance to motion of tubular conveyer. Gorny informatsionno-analitichesky byuleten, (6), 191-198

2. Dmitriev, V. G., Efimov, M. S. (2008). Features of motion of belt of tubular conveyer on the area of route bend in a horizontal plane. Izvestia vuzov. Mining Journal, (3), 99102

3. Efimov, M.C. (2008). The influence of the different factors on angle of deflection of the belt of tubular conveyer. Gorny informatsionno-analitichesky byuleten, (8), 235-237

4. Babakov, I.M. (1968). Moskva: Teoriya kolebaniy. Nauka 